Draft version SePtember 16, 2020

Preprint typeset using $\mathrm{AT}_{\mathrm{E} X} \mathrm{X}$ style emulateapj v. 12/16/11

\title{
UPGRADED GMRT OBSERVATIONS OF THE COMA CLUSTER OF GALAXIES: THE OBSERVATIONS
}

\author{
DHARAM V. LAL \\ National Centre for Radio Astrophysics - Tata Institute of Fundamental Research Post Box 3, Ganeshkhind P.O., Pune 41007, India \\ Draft version September 16, 2020
}

\begin{abstract}
We have used the upgraded Giant Metrewave Radio Telescope to map the Coma cluster of galaxies at 250-500 MHz and 1050-1450 MHz bands. These 6". 26 and 2"' 18 resolutions observations allow detailed radio structures to be determined of all detected radio sources that show both discrete pointlike and extended morphologies. We present images of a subset of 32 brightest $\left(S_{\nu} \gtrsim 30 \mathrm{mJy}\right)$ and dominant sources, and several sources show discrete pointlike radio morphologies. We find the steepening of the spectra consistent with synchrotron cooling in the majority of sources and the median for spectral indices is -0.78 , suggesting that $\sim 59 \%$ sources have steep spectra. The nature and the statistical properties of the radio sources in the Coma cluster will be discussed in subsequent papers.

Subject headings: Active galaxies (17); Radio jets (1347); Radio active galactic nuclei (2134); Coma cluster (270); Radio continuum emission (1340); Galaxy clusters (584)
\end{abstract}

\section{INTRODUCTION}

The largest hierarchical units of the distribution of matter in the universe, called clusters are asymmetric structures, located at the intersecting nodes of the filamentary structure of the cosmic web. They are constantly evolving and growing by accreting matter from the surrounding large-scale structures. Beyond radio sources in galaxy clusters, a fraction of merging clusters host cluster-wide diffuse emission, implying the existence of relativistic electrons and a large-scale magnetic field, called the radio halos. These continue to be the topic of much current research. Therefore, probing the gaseous medium in galaxy clusters through the study of radio emission of "halo sources" reveals important information on physical processes in clusters. Of particular interest is the origin of both the relativistic particles and the associated magnetic field, which are required to generate the observed diffuse synchrotron emission.

The prototype cluster, the Coma cluster of galaxies (Abell 1656; $z=0.0235$ Struble \& Rood 1999) contains more than 30 cluster radio galaxies, of different radio morphologies and of different optical morphological types (Venturi, Giovannini \& Feretti 1990; Waldram et al. 1996; $\quad$ Brown \& Rudnick 2011; $\quad$ Miller. Hornschemeier \& Mobasher 2009; Bonafede et al. 2015). Moreover, the Coma cluster is permeated by giant diffuse radio halo emission (Willson 1970), which is not identified with any individual galaxy and makes the Coma cluster exceptional in the radio domain. In addition, the two dominant galaxies, NGC 4874 (the brightest cluster galaxy, BCG) and NGC 4889 have a velocity difference of $\sim 700 \mathrm{~km} \mathrm{~s}^{-1}$ between them. Taken together with the presence of radio halo, this suggests that the Coma cluster has undergone a major merger (Fitchett \& Webster 1987; Adami et al. 2005; Okabe. Okura \& Futamase 2010).

Neumann et al. (2001) using XMM-Newton further suggests that NGC 4839 group, located towards the south is also merging with the Coma cluster. This nearby cluster is a strong, $\mathrm{X}$-ray bright and hot $(\sim 8$

dharam@ncra.tifr.res.in
$\mathrm{keV}$ ) source (see, e.g. Simionescu et al. 2013, and references therein), which deviates significantly from spherical symmetry (Eckert et al. 2012), and is a dynamically active, non-cool core system (Edge et al. 1990; Vikhlinin. Forman \& Jones 1999; Neumann et al. 2001).

The sources at the center of the Coma cluster, both the BCG and NGC 4874, along with the extended radio halo emission are known collectively as Coma C (Willson 1970). Here, in this paper, the first in the series, we present observations from the upgraded Giant Metrewave Radio Telescope (uGMRT) project targeted at the enigmatic Coma cluster. We aim to make use of the high sensitivity and high resolution of the uGMRT in order to investigate for the first time the detailed radio structures of radio sources in the Coma cluster. Our images have the advantage of high positional accuracy and sensitivity at low radio frequencies.

We assume a $\Lambda$ CDM cosmology with $\Omega_{\mathrm{m}}=0.27, \Omega_{\Lambda}$ $=0.73$, and $H_{0}=70 \mathrm{~km} \mathrm{~s}^{-1} \mathrm{Mpc}^{-1}$. At the redshift of the cluster, $1^{\prime}$ corresponds to $28 \mathrm{kpc}$. Throughout, positions are given in J2000 coordinates. We define spectral index, $\alpha$, as $S_{\nu} \propto \nu^{\alpha}$; where $S_{\nu}$ is the flux density at frequency, $\nu$. We present our observations and data analysis in Sec. 2 and Sec. 3, respectively. We present our observational results in Sec. 4, and we also discuss radio spectra and luminosities (Sec. 4.2). Sec. 5 summarizes our conclusions and future directions.

\section{OBSERVATIONS}

The GMRT (Swarup et al. 1991) has been recently upgraded with a completely new set of receivers at frequencies $<1500 \mathrm{MHz}$. This upgrade provides the telescope (nearly) seamless frequency coverage from 50 to 1500 $\mathrm{MHz}$ (Gupta et al. 2017).

The Coma cluster of galaxies was re-observed (proposal ddtb270) at band-3 (250-500 MHz band) and band-5 $(1050-1450 \mathrm{MHz})$ of the uGMRT because of increased bandwidth (and hence enhanced $(u, v)$ coverage), higher antenna gain, and a lower system temperature of receivers, which all contribute to much improved sensitivity. This enables it to image high resolution structures along with diffuse, low surface brightness extended 
TABLE 1

LOG OF THE OBSERVATIONS

\begin{tabular}{|c|c|c|c|c|c|c|c|}
\hline $\begin{array}{c}\text { Obs_band } \\
(1)\end{array}$ & $\begin{array}{c}\text { Obs. Date } \\
(2) \\
\end{array}$ & $\begin{array}{l}\text { Cal. } \\
(3) \\
\end{array}$ & $\begin{array}{c}\Delta \nu \\
(\mathrm{MHz}) \\
(4)\end{array}$ & No. of Ch. & $\begin{array}{c}\text { tint. } \\
\text { (hour) } \\
(6)\end{array}$ & $\begin{array}{c}\text { FWHM } \\
\left({ }^{\prime \prime} \times{ }^{\prime \prime},{ }^{\circ}\right) \\
(7)\end{array}$ & $\begin{array}{c}\text { RMS } \\
\left(\mu \mathrm{Jy} \text { beam }^{-1}\right) \\
(8)\end{array}$ \\
\hline \multicolumn{8}{|l|}{ uGMRT array } \\
\hline $250-500 \mathrm{MHz}$ & 2017 Apr 28 & $3 \mathrm{C} 286$ & 200 & 4096 & $\sim 1.8$ & $6.65 \times 5.90,76.39$ & 21.1 \\
\hline $1050-1450 \mathrm{MHz}$ & 2017 Apr 26 & $3 \mathrm{C} 286$ & 400 & 8192 & $\sim 1.5$ & $2.43 \times 1.95,69.51$ & 12.7 \\
\hline
\end{tabular}

Note. - Col. 8: RMS noise at the half power point (see also Sec 3 for a discussion).

structures with good angular resolution and with good sensitivity. The time sampling of the data was $2.67 \mathrm{~s}$, which is sufficient to sample the phase fluctuations of the ionosphere and to avoid time smearing for sources at the outer edge of the field of view. The GMRT Wideband Backend was used as the correlator, spanning the frequency range of $300-500 \mathrm{MHz}$ and $1050-1450 \mathrm{MHz}$ at the $250-500 \mathrm{MHz}$ band and the $1050-1450 \mathrm{MHz}$ band, respectively in two polarizations, RR and LL. A summary of the observational setup is detailed in Table 1

\section{DATA REDUCTION}

The data reduction used in this paper follows standard imaging methodologies; however, as uGMRT data reduction is a relatively new process, we summarize below the procedure.

(i) Periodic observations of calibration source 3C 286, once every 30 minutes at the $250-500 \mathrm{MHz}$ band and every 20 minutes at the $1050-1450 \mathrm{MHz}$ band were used to correct for the flux density scale and bandpass shape and to perform phase calibration. The data analysis, including editing of bad data, and gain and bandpass calibrations were carried out using "classic" AIPS.

(ii) We used the default, revised flux density scale for low frequencies using the coefficients for 3C 286 in the AIPS task SETJY

$$
\begin{array}{rl}
\log (S)=1.24 & 81-0.4507 \times \log \left(\nu_{G}\right) \\
& -0.1798 \times\left[\log \left(\nu_{G}\right)\right]^{2} \\
+ & 0.0357 \times\left[\log \left(\nu_{G}\right)\right]^{3} ;
\end{array}
$$

where $S$ is the spectral flux density and $\nu_{G}$ is the frequency in GHz (see also Perley \& Butler 2017).

(iii) After the initial calibration, steps (i) and (ii), the $200 \mathrm{MHz}$ of $300-500 \mathrm{MHz}$ data and the $400 \mathrm{MHz}$ of $1050-1450 \mathrm{MHz}$ data were split into five $40 \mathrm{MHz}$ and eight $50 \mathrm{MHz}$ sub-bands, respectively. These sub-bands were analyzed separately with no averaging of the spectral channels.

(iv) Areas of $\approx 9 \mathrm{deg}^{2}$ and $\approx 1 \mathrm{deg}^{2}$ were imaged for 300-500 MHz data and 1050-1450 MHz data, respectively; they are just bigger than the first null of the primary beam to image sources far from the phase center and to correct for antenna based gains.

(v) A standard self-calibration procedure and the method of peeling were performed in AIPS on each sub-band of 300-500 $\mathrm{MHz}$ data to correct for direction-dependent errors. We aimed to obtain the best possible model of all detected sources in the imaged field. Therefore, we modeled $\approx 100$, both extended and pointlike, radio sources that are (nearly) uniformly distributed, providing us with the gains for each direction in the sky at this band. This peeling step was useful in removing the direction-dependent gains, including the calibration of ionospheric effects, and hence was employed only for 300-500 MHz data; however, only self-calibration procedure was performed for 1050 $1450 \mathrm{MHz}$ data.

(vi) The calibrated data for five $40 \mathrm{MHz}$ and eight $50 \mathrm{MHz}$ sub-bands at the two uGMRT bands were further joined to form full 200 and $400 \mathrm{MHz}$ calibrated visibility data. The combined visibilities were imaged using TCLEAN. In order to ensure image fidelity over the full field and band, we used 3D imaging (gridder = 'widefield'), two Taylor coefficients (nterms $=2$ ), and Briggs weighting (robust $=0.5)$ in task TCLEAN.

(vii) Using the model from (vi), a final amplitude and phase self-calibration with a solution interval equal to length of observation was then carried out using GAINCAL and APPLYCAL tasks in CASA. The two polarizations, $\mathrm{RR}$ and LL were combined to obtain final total intensity image using TCLEAN task in CASA as detailed above in step (vi). We built a task WBPBUGMRT using the coefficients of an eighth order polynomial fit to the antenna primary beam for the different bands of the uGMRT2, similar to WIDEBANDPBCOR task in CASA. It computes Taylor-coefficient images that represent the primary beam shape of the GMRT antennas and applies them to the output images from TCLEAN task to produce primary beam corrected images.

1 First, determine the gains for brightest 5-10 radio sources over the imaged region and subtract these gains from the observed data. Next, image the residual visibilities after flagging whenever needed. These two steps were repeated 10-20 times depending on the frequency of the sub-band, which provided us with the gains for $\sim 100$ directions over the imaged region. In addition to these brightest $\left(S_{\nu} \gtrsim 30 \mathrm{mJy}\right)$ and dominant 32 sources discussed here, we only determined the gains in the directions of strong $\left(S_{\nu} \gtrsim\right.$ $10 \mathrm{mJy}$ ) compact sources. We do not perform these steps for the complex/diffuse weak sources, where too many degrees of freedom might add to the problem, and avoid the problem of overfitting (Bhatnagar et al. 2008).

2 uGMRT primary beams:

www.ncra.tifr.res.in/ncra/gmrt/gmrt-users/observing-help/ugmrt-primar 


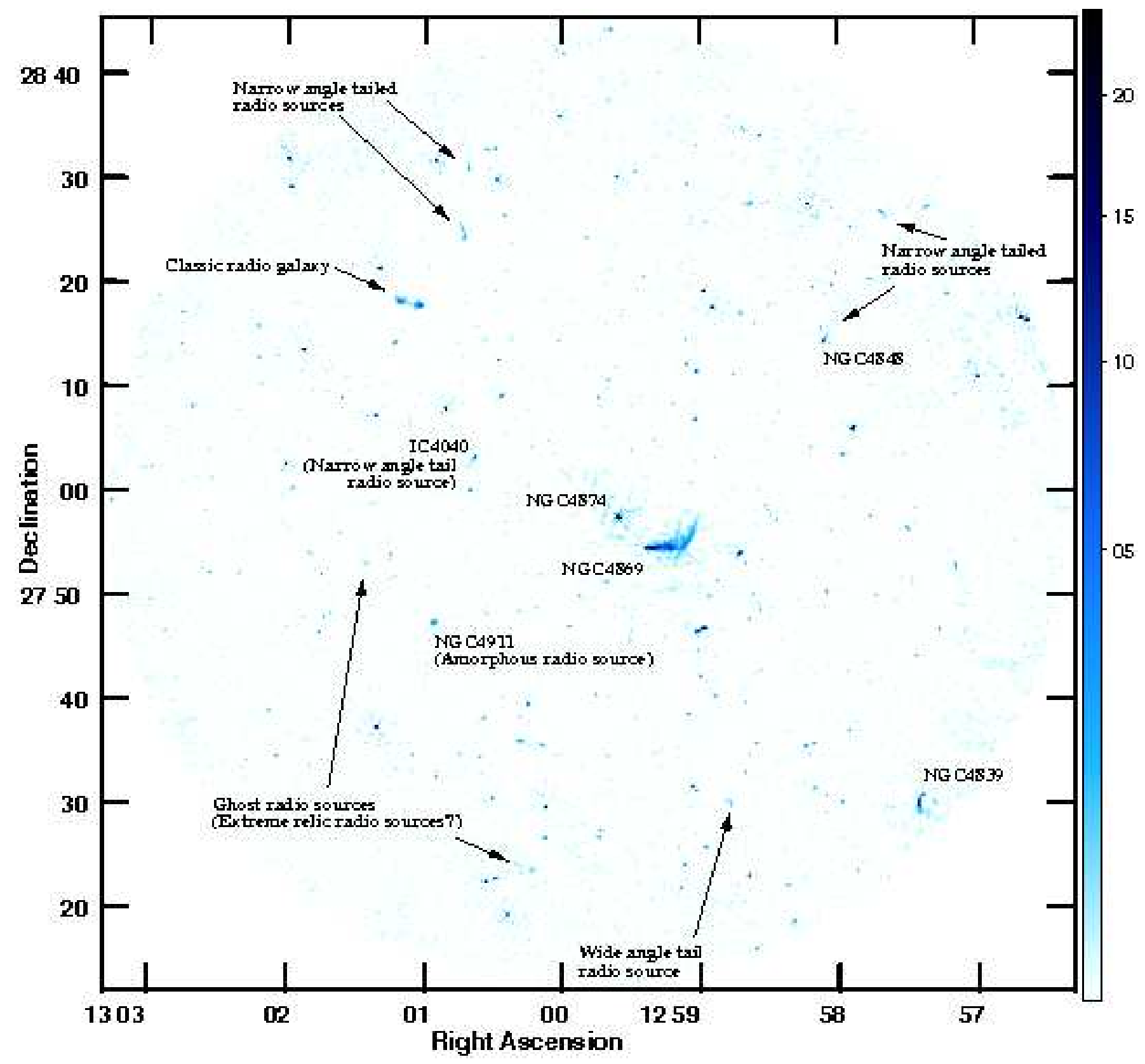

FIG. 1. - Image of the Coma cluster of galaxies at the $250-500 \mathrm{MHz}$ band of the uGMRT. The phase center is RA $=12^{\mathrm{h}} 59^{\mathrm{m}} 29^{\mathrm{s}}$ and decl. $=27^{\mathrm{d}} 55^{\mathrm{m}} 48^{\mathrm{s}}$ and the radius (or the field of view) is $\sim 45^{\prime}$, corresponding to the center frequency, $400 \mathrm{MHz}$. The synthesized beam is $66^{\prime \prime} 65 \times 55^{\prime \prime} 90$ at a P.A. of 76.39 , the peak surface brightness is $114.5 \mathrm{mJy}^{\circ}$ beam $^{-1}$ and the RMs noise is $21 \mu \mathrm{Jy}^{\circ}$ beam $^{-1}$ (see also Sec 3 for a discussion). The vertical gray scale bar is in units of mJy beam ${ }^{-1}$. Representative members of the cluster, including BCG NGC 4874 along with representative radio sources, candidate extreme relic radio sources, etc., are labeled, showing a variety in radio morphologies.

The final images corrected for the primary beam shape of the GMRT antennas for the two bands, shown in Fig.1 and Fig. 2, have an RMS noise of $\approx 21 \mu \mathrm{Jy}_{\text {beam }}{ }^{-1}$ and $\approx 13 \mu \mathrm{Jy}$ beam $^{-1}$ at the half power point and a dynamic range of $\approx 5300$ and $\approx 1700$, respectively. The error in the estimated flux density, both due to calibration and due to systematic, is $\lesssim 4 \%$. The RMS noise is a factor of 1.8-2.1 higher close to the phase center where two dominant radio sources and an extended radio source, NGC 4874, the BCG and NGC 4869, are present. Subsequent analyses of the integrated spectra of all these sources shows that our flux densities measurements are consistent with the literature data (see also Sec. 4.2).

\section{RESULTS}

The large scale radio morphology of the Coma cluster at the $250-500 \mathrm{MHz}$ band and the $1050-1450 \mathrm{MHz}$ band of the uGMRT is shown in Fig. 1 and Fig. 2, respectively. These are the deepest GMRT images after its upgrade (at the $250-500 \mathrm{MHz}$ band) to our knowledge. Fig. 3 shows images of a subset of the 32 brightest $\left(S_{\nu} \gtrsim 30 \mathrm{mJy}\right)$ and dominant sources at the $250-500 \mathrm{MHz}$ band and the majority of these show discrete pointlike radio morphologies. Similarly, Fig. 4 shows images of 5 (out of 32) 


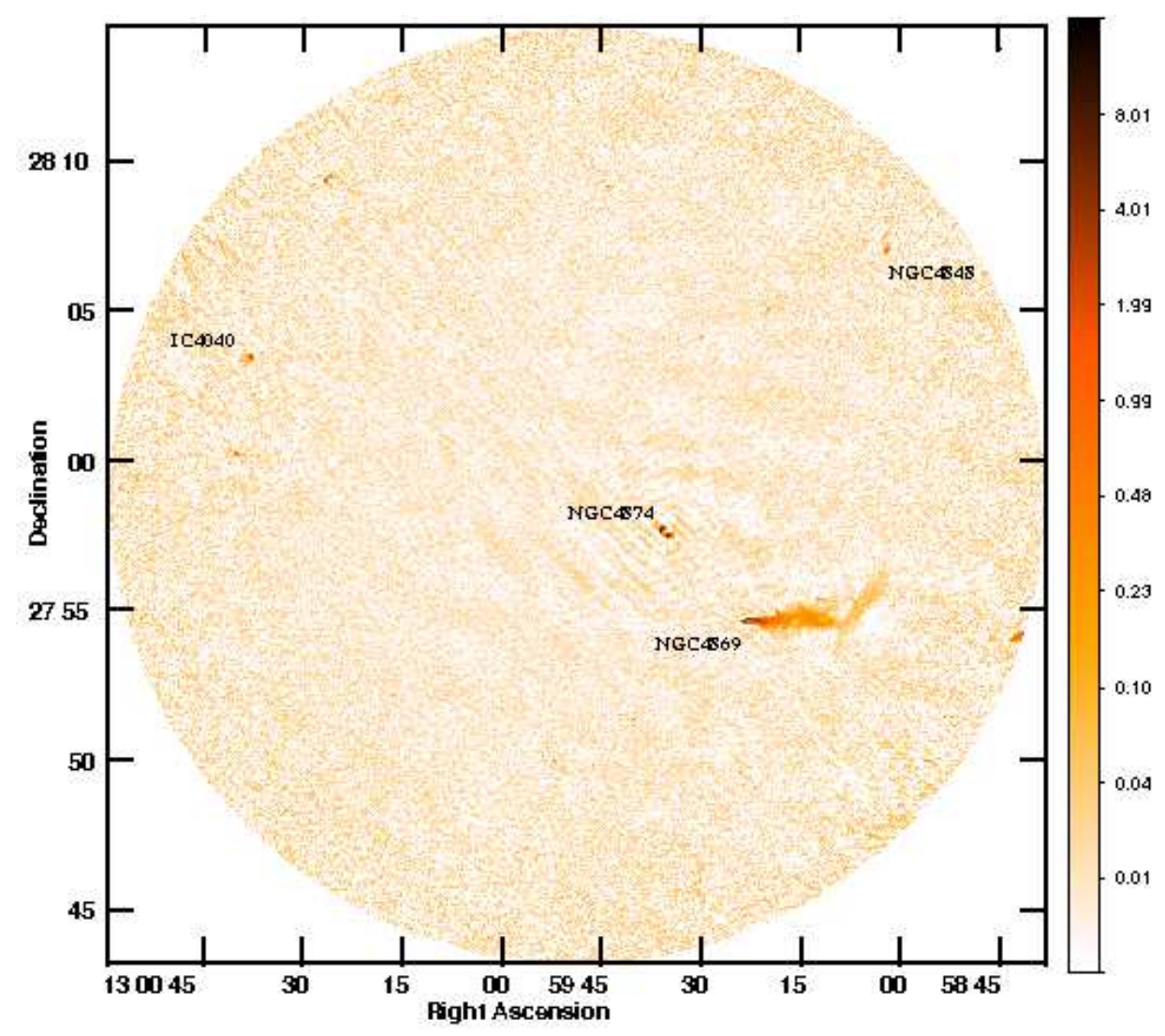

FIG. 2.- Image of the Coma cluster of galaxies at the $1050-1450 \mathrm{MHz}$ band of the uGMRT. The radius (or the field of view) is $\sim 15.6^{\prime}$, corresponding to the center frequency, $1250 \mathrm{MHz}$. The synthesized beam is $2^{\prime \prime} .43 \times 11^{\prime \prime} 95$ at a P.A. of $699^{\circ} 51$, the peak surface brightness is $22.5 \mathrm{mJy}$ beam $^{-1}$ and the RMS noise is $12.7 \mu \mathrm{Jy} \mathrm{beam}^{-1}$ (see also Sec 3 for a discussion). The vertical gray scale bar is in units of mJy beam ${ }^{-1}$.

sources imaged in the $\sim 15 ! 6$ field of view at the 1050 $1450 \mathrm{MHz}$ band. The flux density is determined using AIPS task JMFIT for pointlike, symmetric sources and using AIPS task TVSTAT for all extended (source sizes $\gtrsim$ $15^{\prime \prime}$ ) irregularly shaped radio sources. The error bars in Table 3 are based on the local RMS noise as evaluated in a circle of $5^{\prime}$ in diameter centered on the source position; this is also the error associated with the peak flux density measurement. Table 2 lists these 32 modeled, brightest $\left(S_{\nu} \gtrsim 30 \mathrm{mJy}\right)$, and both pointlike and extended radio sources present in the field of view $\left(90^{\prime} \pm 4^{\prime}\right)$ at $250-500$ $\mathrm{MHz}$ band after correcting for the primary beam shape. The columns are as follows: (1) source ID; (2 and 3) likely radio core position (R.A. and decl.) at $400 \mathrm{MHz}$; (4) redshift of source, from NED; and (5) comments on the source structure at the $250-500 \mathrm{MHz}$ band.

\subsection{Notes on the dominant radio sources}

Salient features of radio morphologies of these brightest $\left(S_{\nu} \gtrsim 30 \mathrm{mJy}\right)$ and dominant radio sources are presented in Table 2. Below we provide additional notes on our interpretation of the radio morphologies for these radio sources in the order presented in Table 2. We also include notes of the radio morphologies for five sources, Source_IDs, 01, 02, 09, 17 and 20, that were imaged at the $1050-1450 \mathrm{MHz}$ band.

01. NGC 4874 - The dominant BCG of the Coma cluster (Baier \& Tiersch 1990) that is located close to the peak of the distributions of galaxies and Xray gas. The optical host is a regular low brightness elliptical galaxy (Capetti et al. 2000) located at $z=0.02394$. It is also a strong radio source with a wide-angle tail (WAT) radio morphology and its projected maximum angular extension is $\sim 30^{\prime \prime}$, corresponding to $\sim 15 \mathrm{kpc}$. We detect the radio core and two radio jets forming a wide angle between them in our uGMRT $250-500 \mathrm{MHz}$ band image.

The source is a dominant $\mathrm{cD}$ radio galaxy, where the local thermal noise is a factor of $\sim 2.1$ higher than at the half power point. In the vicinity of this dominant source, the dynamic range is smallest leading to residual deconvolution (and hence phase calibration) errors $(\lesssim 1 \%)$ at the $1050-1450 \mathrm{MHz}$ band

02. NGC 4869 - Another member of the Coma cluster lies well outside the cluster core: $\sim 40^{\prime}$ away to the southwest of NGC 4874. The radio source is hosted by an elliptical host (Venturi, Giovannini \& Feretti 1990). The structure of this source is typical of a head-tail, narrow angle tail (NAT, e.g., 3C 129: Lal \& Rao 2004) radio source with a weak unresolved radio core, two oppositely directed radio jets, and a long-low surface brightness tail pointing away from the cluster center. The tailed-jet has a conical shape centered on the nucleus and 

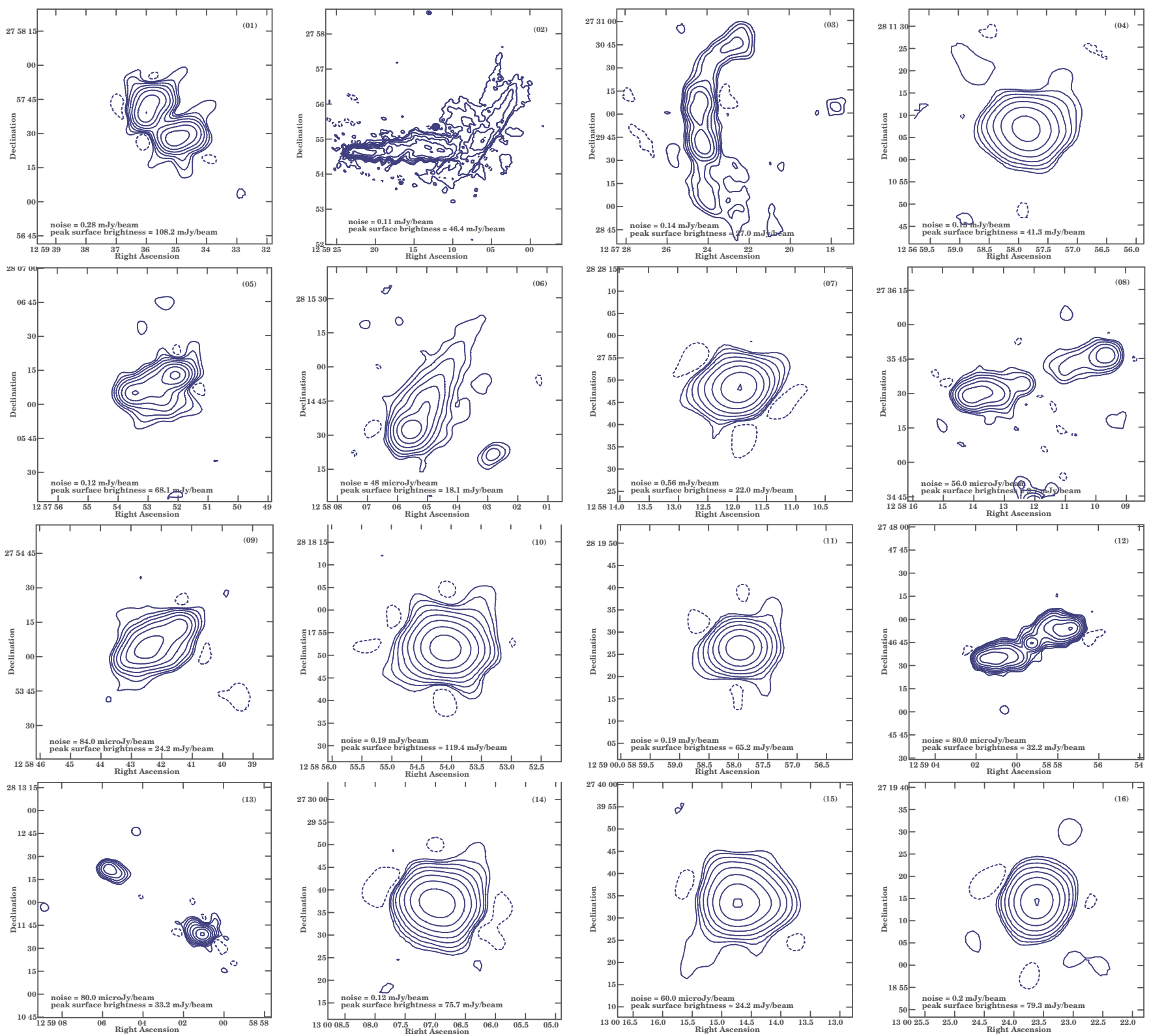

FIG. 3.- Images of brightest $\left(S_{\nu} \gtrsim 30 \mathrm{mJy}\right)$ and dominant, both pointlike and extended, radio sources in the Coma cluster at 250-500 $\mathrm{MHz}$ band of the uGMRT. These radio sources are in the order presented in Table 2 The lowest radio contour plotted is three times the local RMS noise and increasing by factors of 2. The local RMS noise and continuum peak surface brightness of the source are denoted in each panel (lower-left corner) along with its Source_ID (upper-right corner).

it initially expands and then recollimates. Subsequently, the radio jet bends by $\sim 70 \mathrm{deg}$ with respect to the initial direction of propagation. There is also the presence of diffuse extensions toward the inner edge and sharpness in surface brightness toward the outer edge of the radio source, possibly due to the motion of this head-tail radio source around the dark matter potential.

Our $1050-1450 \mathrm{MHz}$ band uGMRT image again shows a head-tail radio source. We detect the weak radio core, with two narrow angle radio jets pointing away from the radio core forming a long tail. The conical shaped jet centered on the nucleus expands, recollimated and then bends by $\sim 70 \mathrm{deg}$ as is seen in our uGMRT 250-500 MHz band image, which is also reported by Feretti et al. (1990). The high surface brightness outer edge is seen, whereas the low surface brightness, diffuse extensions toward the inner edge seen in our low-frequency $250-500 \mathrm{MHz}$ band image are not seen in our high-frequency $1050-1450 \mathrm{MHz}$ band image, probably because of synchrotron cooling (Feretti et al. 1990).

03. NGC 4839 - This is the compact dominant galaxy of the NGC 4839 group and lies in a peripheral Coma cluster region. The optical host is a low/average surface brightness, disk-dominated galaxy (Oemler 1976). It is located at nearly the same $z=0.02456$ of the Coma cluster. A two- 
Lal, D.V.
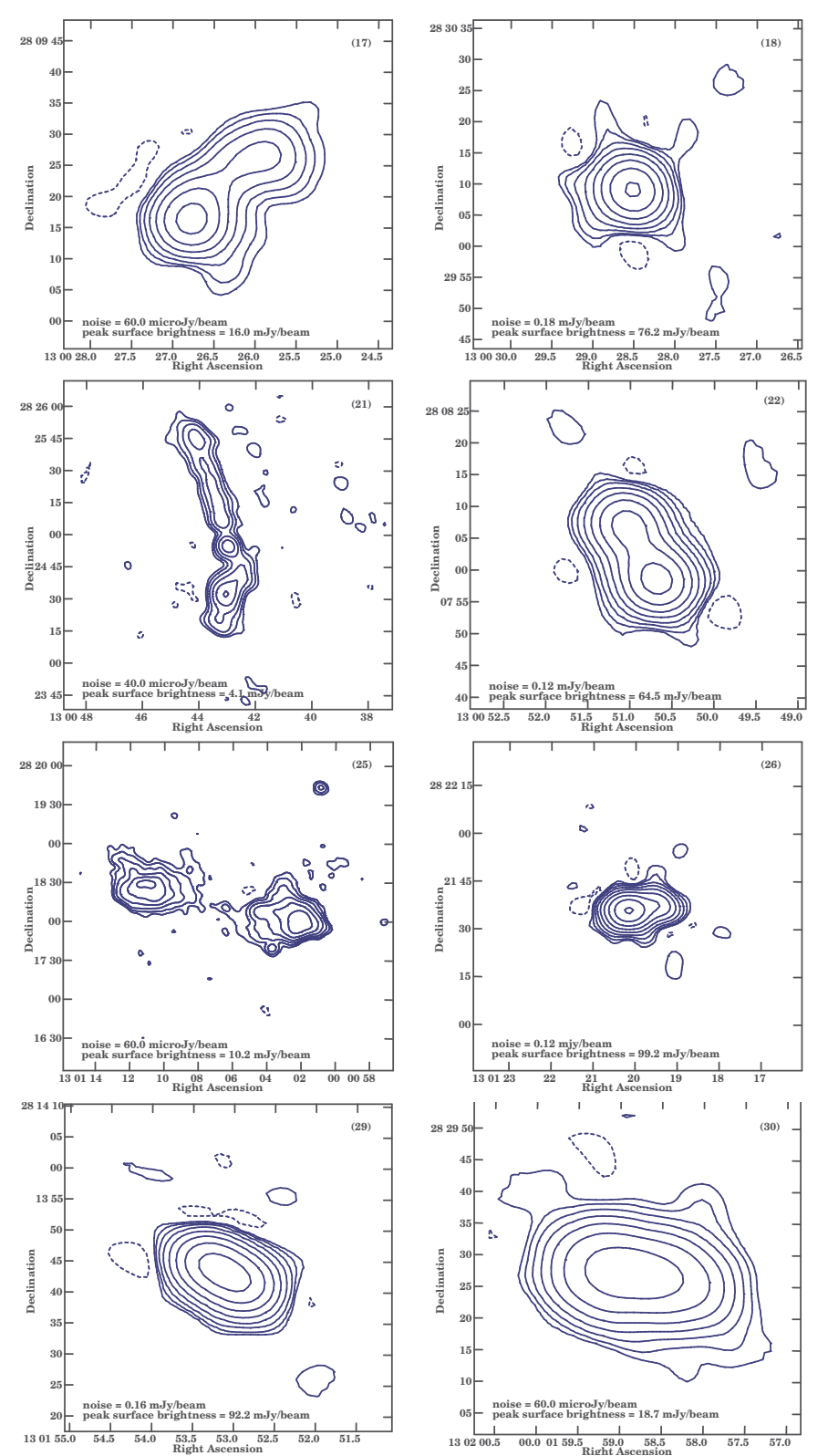

FIG. 3.- Continued
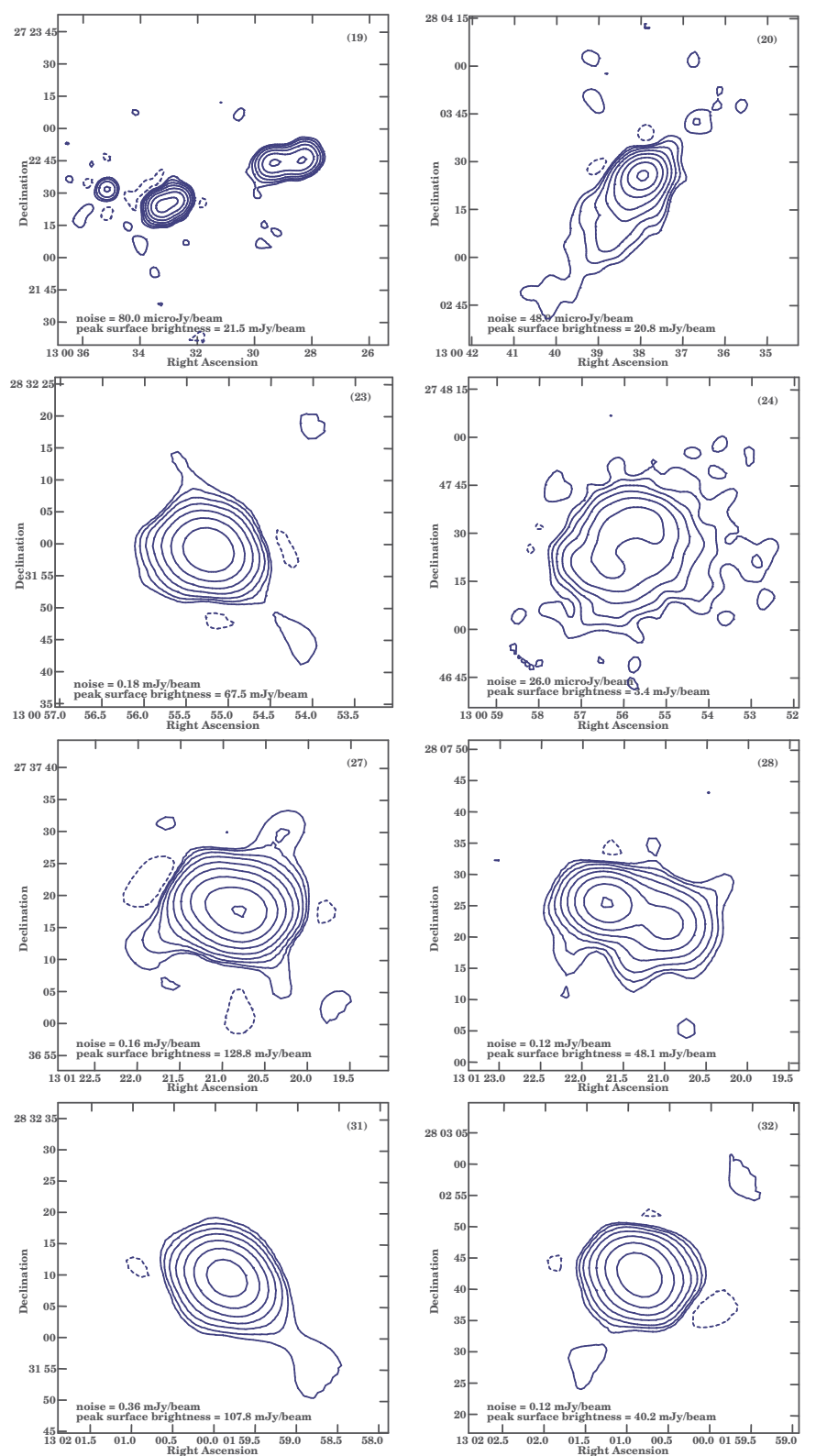

sided radio jet with slight distortions emerging in P.A. $\sim 0^{\circ}$ with a bow shock-like a feature with indications of ram pressure stripping, which suggests that the NGC 4839 group is falling into the Coma cluster (Neumann et al. 2001). The integrated flux density, $S_{408 \mathrm{MHz}}(=316.7 \mathrm{mJy}$; Kim et al. 1994) is a factor of $\sim 1.6$ higher than our measurement. This is because the radio source is also a relic source lying at the edge of the field of view, where our ability to map low surface brightness diffuse emission is poor.

06. NGC 4848 - A head-tail radio source appears to be undergoing ram pressure stripping (see also Chen et al. 2020). The optical host is a blue disk Scd galaxy (Bravo-Alfaro et al. 2000).

09. 5C 4.70 - The uGMRT 1050-1450 MHz band im-

age shows a classic double source FR II radio morphology (Fanaroff \& Riley 1974) consisting of edgebrightened, diffuse lobe emission with clear hot spots at the end of the source.

12. WISEA J125859.32+274644.9 - A classic twosided double radio source of FR II radio morphology. The radio core is well detected.

13. SDSS J125903.80+281145.6 - This again is a classic radio galaxy of FR II radio morphology, though the southwest radio lobe is associated with $5 \mathrm{C} 4.77$ radio source.

14. WISEA J130006.87+272936.8 - An unresolved radio source, which is $\sim 4^{\prime \prime}$ away from Abell 1656:[EF2011] 1595 (NED classification) source. This NED classified source has a photometric redshift of 0.22001 . 

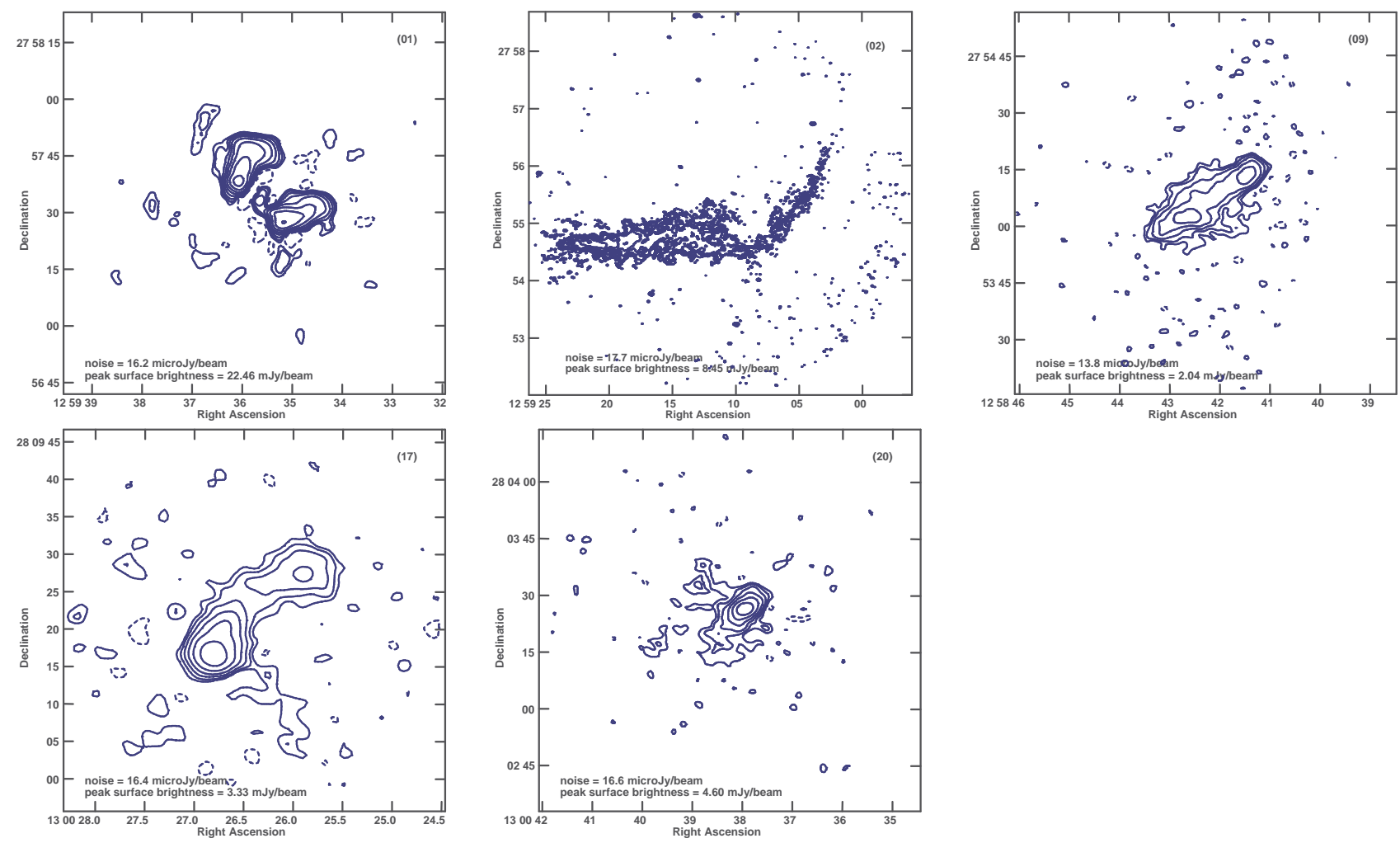

FIG. 4.- Images of the five of 32 brightest $\left(S_{\nu} \gtrsim 30 \mathrm{mJy}\right)$ and dominant, both pointlike and extended radio sources in the Coma cluster detected in a small field of view at the 1050-1450 MHz band of the uGMRT. These radio sources are in the order presented in Table 2 The lowest radio contour plotted is three times the local RMS noise and increasing by factors of 2 . The local RMS noise and continuum peak surface brightness of the source are denoted in each panel (lower-left corner) along with its Source_ID (upper-right corner).

17. WISEA J130026.68+280920.0 - The uGMRT 1050-1450 MHz band image shows a double source of FR II radio morphology, which consists of an edge-brightened, diffuse radio lobe emission with clear hot spots at the end of the source. The two jets have bent after emanating from the radio core, probably because of ram pressure.

19. SDSS J130030.76+272239.4 - We have classified it to be a triple source because the 2.5 times the RMS noise contour shows three bright peaks as a single connected contiguous radio source and the SDSS optical source is located at the center. If our understanding is correct, it is possibly a WAT radio galaxy.

20. IC 4040 - A NAT radio source hosted by CGCG 160-252 galaxy of spiral or an irregular morphological type. It appears to be undergoing ram pressure stripping (see also Chen et al. 2020).

This NAT source does not show a similar extent in the $1050-1450 \mathrm{MHz}$ band image as compared to the $250-500 \mathrm{MHz}$ band image, probably because of synchrotron cooling.

21. WISEA J130043.86+282458.8 - A WAT radio source with a radio core at the center, which coincides with the WISE source. The northern radio jet seems to be moving straight, whereas the southern jet has been bent. The radio morphology also suggests it to be of a "hybrid", with the northern jet to be of FRI type and southern jet to be of FR II type. Two jets show varying projected radio sizes, but the southern jet may have encountered more cluster gas, and hence the ram pressure, as compared to the northern jet. The flux density at $1400 \mathrm{MHz}=9.4 \pm 0.2 \mathrm{mJy}$ (corresponds to C1A-923; Miller, Hornschemeier \& Mobasher 2009), which makes spectral index $\alpha(400-1400$ $\mathrm{MHz})=-0.96 \pm 0.02$. The apparent steep spectrum could possibly be due to extended emission from this WAT source that is resolved at high frequency. (Miller, Hornschemeier \& Mobasher 2009) report that the source deblends with $\mathrm{C} 1 \mathrm{~A}-922$ and possibly adjoins $\mathrm{C} 1 \mathrm{~A}-152, \mathrm{C} 1 \mathrm{~A}-153$, and C1A-156 as well. Condon et al. (1998) note $S_{1400 \mathrm{MHz}}=9.2 \pm 1.1 \mathrm{mJy}$ (the NVSS survey image), which is consistent with flux density of C1A-923 (Miller. Hornschemeier \& Mobasher 2009).

23. SDSS J130055.21+283205.7 - An unresolved radio source, which is $\sim 9^{\prime \prime}$ away from the WISEA J130055.69+283200.8 (NED classification) source. This NED classified WISE source has a photometric redshift of 0.25737 .

24. NGC 4911 - The host galaxy is a very bright giant early spiral of an $\mathrm{Sb}$ morphological type. The source is possibly interacting with its neighbor DRCG 27-62 (Bravo-Alfaro et al. 2000).

25. WISEA J130106.38+281813.0 - A classical twosided radio galaxy showing double lobe FR II mor- 
TABLE 2

A Short summary of the nature of dominant sources in the Coma Cluster field at 400 MHz USING the uGMRT.

\begin{tabular}{|c|c|c|c|c|c|}
\hline & \multirow{2}{*}{$\begin{array}{l}\text { Source ID } \\
(1)\end{array}$} & \multicolumn{2}{|c|}{ Position at $400 \mathrm{MHz}$} & \multirow{2}{*}{$\begin{array}{l}z \\
(4) \\
\end{array}$} & \multirow{2}{*}{$\begin{array}{l}\text { Salient feature of radio morphology } \\
(5)\end{array}$} \\
\hline & & $\begin{array}{c}\text { R.A. } \\
(2)\end{array}$ & $\begin{array}{c}\text { Decl. } \\
(3)\end{array}$ & & \\
\hline 01. & NGC 4874 & $12: 59: 35.71$ & $+27: 57: 33.37$ & 0.02394 & The BCG of Coma cluster; $5 \mathrm{C} 4.85, \mathrm{C} 1 \mathrm{~B}-127$ \\
\hline 02 . & NGC 4869 & $12: 59: 23.36$ & $+27: 54: 41.73$ & 0.02288 & An NAT radio source; $5 \mathrm{C} 4.81, \mathrm{C} 1 \mathrm{~B}-903$ \\
\hline 03. & NGC 4839 & $12: 57: 24.36$ & $+27: 29: 52.14$ & 0.02456 & The cD galaxy of NGC 4839 group; 5C $4.51, \mathrm{C} 1 \mathrm{C}-904$ \\
\hline 04. & WISEA J125656.53+281101.4 & $12: 56: 57.84$ & $+28: 11: 07.32$ & & A discrete point source that is $\sim 19^{\prime \prime}$ away from $5 \mathrm{C} 4.45$ \\
\hline 05 . & WISEA J125752.93+280609.4 & $12: 57: 52.74$ & $+28: 06: 08.26$ & 0.45360 & An FR II radio galaxy; $5 \mathrm{C} 4.54, \mathrm{C} 1 \mathrm{~B}-012$ \\
\hline 06. & NGC 4848 & $12: 58: 05.54$ & $+28: 14: 32.30$ & 0.02351 & An NAT radio source; $5 \mathrm{C} 4.58, \mathrm{C} 1 \mathrm{~A}-020 \& \mathrm{C} 1 \mathrm{~A}-021$ \\
\hline 07. & SDSS J125811.97+282748.6 & $12: 58: 12.00$ & $+28: 27: 48.87$ & 3.17688 & An unresolved radio source; $\mathrm{C} 1 \mathrm{~A}-026$ \\
\hline 08. & WISEA J125812.36+273534.7 & $12: 58: 12.26$ & $+27: 35: 34.11$ & 0.44673 & An FR II radio galaxy; $\mathrm{C} 1 \mathrm{C}-042, \mathrm{C} 1 \mathrm{C}-047 \& \mathrm{C} 1 \mathrm{C}-049$ \\
\hline 09. & $5 \mathrm{C} 4.70$ & $12: 58: 42.24$ & $+27: 54: 06.17$ & & A double radio source; $\mathrm{C} 1 \mathrm{~B}-065$ \\
\hline 10 . & WISEA J125854.14+281752.3 & $12: 58: 54.12$ & $+28: 17: 52.78$ & 0.38160 & Point-like, unresolved radio source; $\mathrm{C} 1 \mathrm{~A}-056$ \\
\hline 11. & WISEA J125858.10+281924.6 & $12: 58: 58.08$ & $+28: 19: 27.41$ & & A discrete point source; $5 \mathrm{C} 4.75, \mathrm{C} 1 \mathrm{~A}-060$ \\
\hline 12. & WISEA J125859.32+274644.9 & $12: 58: 59.52$ & $+27: 46: 45.00$ & 0.02300 & An FR II radio galaxy; $5 \mathrm{C} 4.74, \mathrm{C} 1 \mathrm{C}-61$ \\
\hline 13. & SDSS J125903.80+281145.6 & $12: 59: 03.43$ & $+28: 12: 01.46$ & & An FR II radio galaxy; $\mathrm{C} 1 \mathrm{~A}-061$ \\
\hline 14. & WISEA J130006.87+272936.8 & $13: 00: 06.84$ & $+27: 29: 37.33$ & & An unresolved source; C1C-151 \\
\hline 15 . & SDSS J130014.76+273932.8 & $13: 00: 14.76$ & $+27: 39: 34.39$ & 0.37984 & Amorphous, high surface brightness radio source \\
\hline 16. & NVSS J130023+271915 & $13: 00: 23.76$ & $+27: 19: 14.51$ & & A discrete point and an unresolved source; $5 \mathrm{C} 4.97$ \\
\hline 17. & WISEA J130026.68+280920.0 & $13: 00: 26.35$ & $+28: 09: 21.74$ & & A double radio source; $5 \mathrm{C} 4.102, \mathrm{C} 1 \mathrm{~B}-187$ \\
\hline 18. & FBQS J1300+2830 & $13: 00: 28.44$ & $+28: 30: 10.34$ & 0.64709 & A pointlike radio quasar source; $5 \mathrm{C} 4.105, \mathrm{C} 1 \mathrm{~A}-138$ \\
\hline 19. & SDSS J130030.76+272239.4 & $13: 00: 31.12$ & $+27: 22: 36.31$ & & A bent wide-angle (triple) radio source \\
\hline 20. & IC 4040 & $13: 00: 38.16$ & $+28: 03: 24.25$ & 0.02615 & An NAT radio source; $5 \mathrm{C} 4.108, \mathrm{C} 1 \mathrm{~B}-904$ \\
\hline 21. & WISEA J130043.86+282458.8 & $13: 00: 42.98$ & $+28: 24: 55.23$ & 0.02095 & $\begin{array}{l}\text { A WAT radio source; } 5 \mathrm{C} 4.113, \mathrm{C} 1 \mathrm{~A}-152, \mathrm{C} 1 \mathrm{~A}-153, \mathrm{C} 1 \mathrm{~A}-156 \text {, } \\
\mathrm{C} 1 \mathrm{~A}-922 \& \mathrm{C} 1 \mathrm{~A}-923\end{array}$ \\
\hline 22. & WISEA J130050.84+280803.6 & 13:00:50.88 & $+28: 08: 04.07$ & & A double radio source with core-jet morphology; 5C 4.114 \\
\hline 23. & SDSS J130055.21+283205.7 & $13: 00: 55.08$ & $+28: 32: 00.41$ & & A discrete point source \\
\hline 24 . & NGC 4911 & $13: 00: 56.08$ & $+27: 47: 27.02$ & 0.02663 & An amorphous, diffuse radio source; 5C 4.117, C1B-901 \\
\hline 25. & WISEA J130106.38+281813.0 & $13: 01: 06.27$ & $+28: 18: 11.57$ & 0.37339 & A radio galaxy; $5 \mathrm{C} 4.122, \mathrm{C} 1 \mathrm{~A}-169, \mathrm{C} 1 \mathrm{~A}-910$ \& $\mathrm{C} 1 \mathrm{~A}-911$ \\
\hline 26. & [HB89] $1258+286 \mathrm{NED} 02$ & 13:01:19.92 & $+28: 21: 37.58$ & 1.37176 & A pointlike radio source; $5 \mathrm{C} 4.127, \mathrm{C} 1 \mathrm{~A}-177 \& \mathrm{C} 1 \mathrm{~A}-178$ \\
\hline 27. & WISEA J130120.88+273718.4 & $13: 01: 21.00$ & $+27: 37: 18.56$ & & An unresolved source; 5C 4.128, C1C-205 \& C1C-206 \\
\hline 28. & WISEA J130121.84+280726.7 & $13: 01: 21.36$ & $+28: 07: 25.62$ & 2.26500 & A double radio source; $5 \mathrm{C} 4.129, \mathrm{C} 1 \mathrm{~B}-244 \& \mathrm{C} 1 \mathrm{~B}-245$ \\
\hline 29. & WISEA J130153.05+281344.0 & $13: 01: 53.04$ & $+28: 13: 44.49$ & & An unresolved source; $5 \mathrm{C} 4.134, \mathrm{C} 1 \mathrm{~A}-187 \& \mathrm{C} 1 \mathrm{~A}-188$ \\
\hline 30. & WISEA J130158.70+282928.7 & $13: 01: 58.80$ & $+28: 29: 28.56$ & & A pointlike dumbbell radio source; $5 \mathrm{C} 4.137$ \\
\hline 31. & WISEA J130159.77+283210.1 & $13: 01: 59.88$ & $+28: 32: 11.14$ & & A pointlike source with an extension; $5 \mathrm{C} 4.138$ \\
\hline 32. & WISEA J130200.78+280247.3 & 13:02:00.96 & $+28: 02: 43.45$ & & A discrete pointx and an, unresolved source; $5 \mathrm{C} 4.140, \mathrm{C} 1 \mathrm{~B}-276$ \\
\hline
\end{tabular}

Note. - Notes on our interpretation of the radio morphologies for these brightest and dominant sources are discussed in Sec. 4.1 Col. 1: Source name as identified in the NED.

Col. 2 and 3: The R.A. and decl. (J2000); barring first three sources, the rest of the sources are ordered by the increasing R.A. Source position is given by the brightest component for the unresolved source, whereas for the radio double or the diffuse source, the position is given by the possible radio core.

Col. 4: spectroscopic redshift of source.

Col. 5: a comment on the morphology of the radio source at the $250-500 \mathrm{MHz}$ band. Wherever possible, we also list their $5 \mathrm{C}$ (fifth Cambridge; Willson 1970) survey catalog names and unique 'C1A/B/C' source IDs that are reported in Miller, Hornschemeier \& Mobasher (2009).

phology. The radio lobe toward the west seems to be approaching us since the unresolved radio core and the associated jet are linked to it.

26. [HB89] 1258+286 NED02 - A marginally resolved flat spectrum $(\alpha=-0.44 \pm 0.03)$ quasar source. In addition to a bright nucleus, the source shows corejet morphology or a weak extension in the east-west direction. It is a background source and not part of the Coma cluster of galaxies.

30. WISEA J130158.70+282928.7 - A marginally resolved compact radio source. It seems that the integrated flux density, $S_{408 \mathrm{MHz}}(=242.9 \mathrm{mJy}$; Kim et al. 1994) is a factor of $\sim 3.2$ higher than our measurement. The Kim et al. (1994) measurement includes flux densities for 5C 4.137 (Source_ID 30) and for 5C 4.138 (Source_ID 31) sources, and hence their combined measurement is consistent within errors with our measurement for these two sources.

31. WISEA J130159.77+283210.1 - An unresolved radio source with a possible extension toward the southeast. As stated above (see notes on Source_ID 30), the Kim et al. (1994) measurement is consistent within errors with our measurement.

\subsection{Integrated radio spectra and luminosities}

We made comparisons of our measurements to earlier measurements, which provides a good check of our calibration. Table 3 (Cols. 2-7) lists integrated flux densities at frequencies along with error bars in the measurements and hence the integrated spectra (Col. 8, see also Fig. (5) for 32 brightest and dominant, both pointlike and extended, radio sources. Qualitatively, the radio spectra are classified as (i) straight; (ii) curved, both concave and convex; and (iii) complex (Laing\& Peacock 1980). The two mechanisms that are most likely to produce curvature in the spectrum of radiation from an electron energy distribution initially of the power-law form are (e.g. Miley 1980): (i) synchrotron energy losses, which cause downward curvature at high frequencies, and (ii) selfabsorption of radiation from regions of high brightness temperatures, e.g, compact sources. We see in Fig. 5 that within the errors, all our uGMRT measurements and data from the literature fall nearly along with a 'straight' power law with some hint of energy losses from synchrotron cooling. This suggests that our flux densities measurements are consistent with the literature data, providing confidence in our data calibration and its reduction methodologies.

Almost all sources, except FBQS J1300+2830 
TABLE 3

The total INTENSITY AND SPECTRAL INDEX FOR ALL DOMINANT SOURCES THE COMA CLUSTER FIELD ALONG WITH RADIO LUMINOSITY FOR 17 SOURCES With KNOWN SPECTROSCOPIC REDSHIFTS.

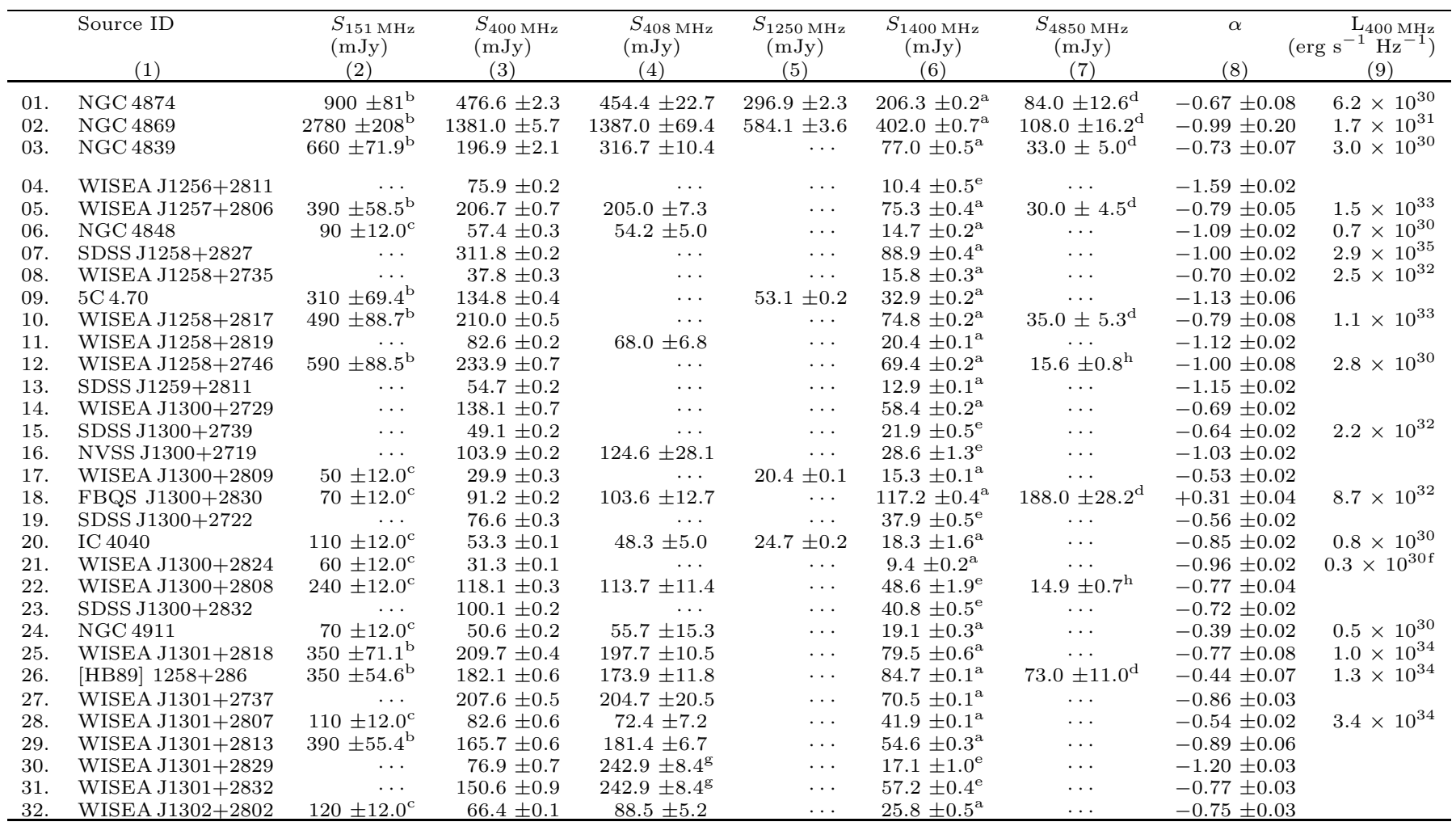

Note. - The integrated flux densities quoted are in mJy along with corresponding error bars when available. The flux densities of both extended and pointlike dominant $\left(S_{\nu} \gtrsim 30 \mathrm{mJy}\right.$ ) radio sources at $400 \mathrm{MHz}$ (Col. 3) and at $1250 \mathrm{MHz}$ (Col. 5) are reported. The flux density is determined either using AIPS task TVSTAT for irregular shaped radio source or using AIPS task JMFIT for our 250-500 MHz band and 1050-1450 band data. The error-bar on the measurement of flux density is based on the local RMs noise as evaluated in a circle of $5^{\prime}$ in diameter centered on the source position. Consequently, in the vicinity of strong sources, including the sources listed here, the local RMS noise was sometimes higher than the RMS noise in empty regions. The measurements at $408 \mathrm{MHz}$ (Col. 4) are from Kim et al. (1994). The rest of the measurements at $151 \mathrm{MHz}$ (Col. 2), $1400 \mathrm{MHz}$ (Col. 6) and $4850 \mathrm{MHz}$ (Col. 7) are from NED. The spectral index (Col. 8) correspond to line representing the best fitting regression to this data. The radio luminosity (Col. 9) is evaluated at $400 \mathrm{MHz}$ for 16 sources that have known spectroscopic redshift (see Table 2).

References: The references for flux density measurements from NED and related notes are coded as follows: (a) Miller, Hornschemeier \& Mobasher $(2009)$ and their radio images have sensitivities ranging from 39 to $45 \mu \mathrm{Jy}$ per $4 . .^{\prime \prime} 4$ beam; (b) Waldram et al. (1996) and they quote an error of about $10 \%$ or less in flux densities; (c) Cordev (1985) and the final map has an RMs level of $12 \mathrm{mJy}^{\mathrm{beam}}{ }^{-1}$; (d) Becker, White \& Edwards (1991) and they report an uncertainty of $\sim 4-6 \mathrm{mJy}$ to the derived flux density of a source; (e) Condon et al. (1998) and they state that RMS fluctuation level is $0.45 \mathrm{mJy}$ beam $^{-1}$ for total intensity images; (f) see Sec. 4.1 for a discussion; (g) the measurement 242.9 mJy includes flux densities for two sources, Source_IDs 30 and 31 (see also Sec. 4.1 for a discussion; Kim et al. 1994), and they report noise on their maps is 8 mJy beam ${ }^{-1}$; (h) Bonafede et al. (2010) and they quote calibration error on the measured flux densities of $\sim 5 \%$.

(Source_ID 18), show steep spectra, an effect attributed to the presence of extended diffuse structures. The spectra for total flux density via. best-fitting regression to data for all sources is reported in Table 3. (Col. 8). The mean and median of these sources are -0.83 and -0.78 , respectively, suggesting that $\sim 59 \%$ sources have steep spectra $(\alpha<-0.7)$. Of the sources, seven sources have relatively steep spectra $(-1.0>\alpha$ $>-1.3)$ and one source has a very steep spectrum $(\alpha$ $<-1.3)$. This very steep spectrum source, WISEA J125752.93+280609.4 (Source_ID 04), $\alpha=-1.59 \pm 0.02$ could be a possible candidate for a high-redshift radio galaxy (Saxena et al. 2018). The infrared colors are not known to deviate from the predicted relations with redshift for a standard giant elliptical galaxy spectrum. The source (WISEA J125752.93+280609.4) is a $13.81 \mathrm{mag}$ source in $K s$ (NED: 2MASS extended objects, final release), makes it unlikely to be a high-redshift radio galaxy from the $K-z$ relation for radio galaxies (Lilly \& Longair 1984).
Of the sources, 17 sources have known spectroscopic redshifts (Table 2). Luminosities were evaluated at $400 \mathrm{MHz}$ for the radio emission (Col. 8, Table 3). This frequency was chosen because very accurate flux densities are available, and contributions from compact cores are small. The radio powers have been $K$-corrected to the rest frame of each source (Lal \& Ho 2010).

\section{CONCLUSIONS}

In this first paper of the series, we have presented in the preceding sections details of the observations, data reduction, and performance assessment for the 250-500 $\mathrm{MHz}$ band and the $1050-1450 \mathrm{MHz}$ band using uGMRT for the Coma cluster of galaxies. An image of a single field of the $1.75 \mathrm{deg}^{2}$ and $0.21 \mathrm{deg}^{2}$ areas have been presented up to the RMS noises of $\approx 21 \mu \mathrm{Jy} \mathrm{beam}^{-1}$ and $\approx 13 \mu \mathrm{Jy}$ beam $^{-1}$ with 6.13 and $2 . .2$ angular resolutions at the $250-500 \mathrm{MHz}$ band and the $1050-1450 \mathrm{MHz}$ band, respectively, representing the deepest uGMRT image at the 250-500 MHz band. We also provide descriptions of radio morphologies and spectra of 32 brightest and dom- 


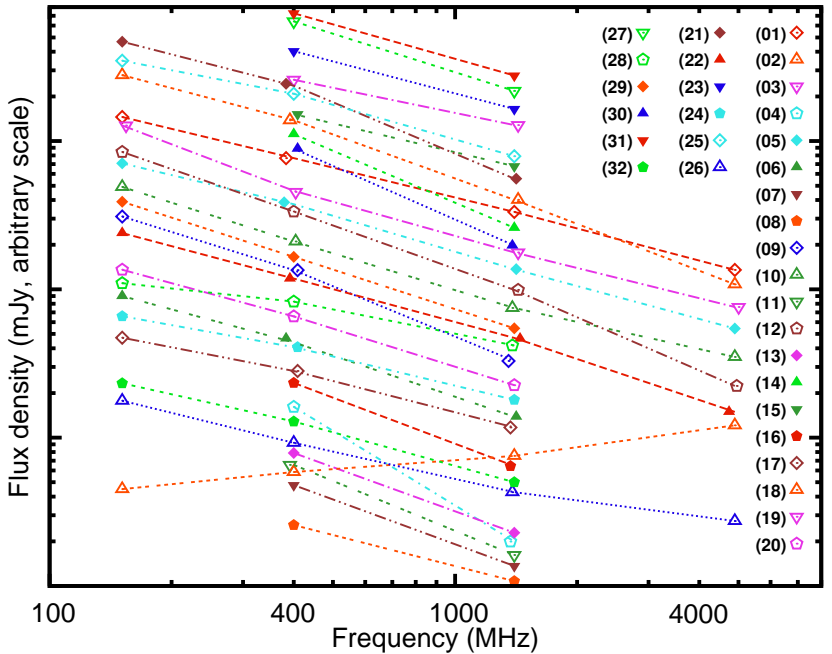

FIG. 5.- Integrated flux densities of 32 brightest and dominant, both pointlike and extended, radio sources in the Coma cluster at the $250-500 \mathrm{MHz}$ band of the uGMRT; data at other frequencies are from the literature (see Table 3 for references). The $408 \mathrm{MHz}$ data (Kim et al. 1994) and our 1050-1450 MHz data are not plotted to avoid overcrowding. The uGMRT flux density measurements are in good agreement with the data. The spectra (and some of the data points) are shifted with respect to one another for clarity. inant, both pointlike and extended, radio sources in the field.

We are undertaking an uGMRT study at 125-250 $\mathrm{MHz}$ (band-2), 250-500 MHz (band-3), and 550-850 $\mathrm{MHz}$ (band-4) of several clusters to make precise flux density measurements of all detected radio sources and model them, thereby aiming to provide exact estimates of the radio halo emission and statistics. The data presented here along with a wealth of multiwavelength data available is being used to investigate the head-tail radio galaxy, NGC 4869 (Source_ID 02, which will form paper II of this series), to study radio luminosity function, to build a sample of ultra-steep spectrum sources, etc.

D.V.L. thanks the anonymous referee for his/her contributions that considerably improved the manuscript. He also thanks Tiziana Venturi and Dave Green for useful discussions, Ishwara-Chandra C.H. for discussions on some aspects of this project, and Sushan Konar for careful reading of the manuscript. He acknowledges the support of the Department of Atomic Energy, Government of India, under project No. 12-R\&D-TFR-5.02-0700. We thank the staff of the GMRT who made these observations possible. The GMRT is run by the National Centre for Radio Astrophysics of the Tata Institute of Fundamental Research. This research has made use of the NED, which is operated by the Jet Propulsion Laboratory, Caltech, under contract with the NASA, and NASA's Astrophysics Data System.

\section{REFERENCES}

Adami, C., Biviano, A., Durret, F. \& Mazure, A. 2005, A\&A, 443, 17

Baier, F. W. \& Tiersch, H. 1990, In Proceedings of the ESO Workshop on Extranuclear Activity in Galaxies, Editors, E. J. A. Meurs \& R. A. E. Fosbury, Garching, bei Munchen: European Southern Observatory, P. 111

Becker, R. H., White, R. L. \& Edwards, A. L. 1991, ApJS, 75, 1 Bhatnagar, S., Cornwell, T. J., Golap, K. \& Uson, J. M. 2008, A\&A, 487, 419

Bonafede, A. 2015, In Proceedings of The many facets of extragalactic radio surveys: towards new scientific challenges, Bologna, PoS (EXTRA-RADSUR2015), P. 63.

Bonafede, A., Feretti, L., Murgia, M., et al. 2010, A\&A, 513, A30

Bravo-Alfaro, H., Cayatte, V., van Gorkom, J. H. \& Balkowski, C. 2000 , AJ, 119, 580

Brown, S. \& Rudnick, L. 2011, MNRAS, 412, 2

Capetti, A., de Ruiter, H. R., Fanti, et al. 2000, A\&A, 362, 871

Chen, H., Sun, M., Yagi, M. et al. 2020, MNRAS, 496, 4654

Condon, J. J., Cotton, W. D., Greisen, E. W., et al. 1998, AJ, 115,1693

Cordey, R. A. 1985, MNRAS, 215, 437

Eckert, D., Vazza, F., Ettori, S., et al. 2012, A\&A, 541, A57

Edge, A. C., Stewart, G. C., Fabian, A. C. \& Arnaud, K. A. 1990 , MNRAS, 245, 559

Fanaroff B. L. \& Riley J. M., 1974, MNRAS, 167, 31

Feretti, L., Dallacasa, D., Giovannini, G. \& Venturi, T. 1990, A\&A, 232, 337

Fitchett, M. \& Webster, R. 1987, ApJ, 317, 653

Gupta, Y., Ajithkumar, B., Kale, H., et al. 2017, Current Science, 113,707
Kim, K.-T., Kronberg, P. P., Dewdney, P. E. \& Landecker, T. L. 1994, ApJS, 105, 385

Laing, R. A. \& Peacock, J. A. 1980, MNRAS, 190, 903

Lal, D. V. \& Ho, L. C. 2010, AJ, 139, 1089

Lal, D. V. \& Rao, A. P. 2004, A\&A, 420, 491

Lilly, S. J. \& Longair, M. S. 1984, MNRAS, 211, 833

Miley, G. K. 1980, ARA\&A, 18, 165

Miller, N. A., Hornschemeier, A. E. \& Mobasher, B. 2009, AJ, 137,4436

Neumann, D. M., Arnaud, M., Gastaud, R., et al. 2001, A\&A, $365, \mathrm{~L} 74$

Oemler, A. 1976, ApJ, 209, 693

Okabe, N., Okura, Y. \& Futamase, T. 2010, ApJ, 713, 291

Perley, R. A. \& Butler, B. J. 2017, ApJS 230, 7

Saxena, A., Marinello, M., Overzier, R. A., et al. 2018, MNRAS, 480,2733

Simionescu, A., Werner, N., Urban, O., et al. 2013, ApJ, 775, 4

Struble, M. F. \& Rood, H. J. 1999, ApJS, 125, 35

Swarup, G., Ananthakrishnan, S., Kapahi, V. K., et al. 1991, Current Science, 60, 95

Venturi, T., Giovannini, G. \& Feretti, L. 1990, AJ, 99, 1381

Vikhlinin, A., Forman, W. R. \& Jones, C. 1999, ApJ, 525, 47

Waldram, E. M., Yates, J. A., Riley, J. M. \& Warner, P. J. 1996, MNRAS, 282, 779

Willson, M. A. G. 1970, MNRAS, 151, 1 